\title{
Anaerobic biodegradation of chloroform and dichloromethane with a Dehalobacter enrichment culture
}

\section{Running Title:}

Biodegradation of chloroform and dichloromethane

\author{
Hao Wang ${ }^{\mathrm{a}}$, Rong $\mathrm{Yu}^{\mathrm{b}}$, Jennifer Webb ${ }^{\mathrm{c}}$, Peter Dollar ${ }^{\mathrm{d}}$, David L. Freedman a,* \\ ${ }^{a}$ Department of Environmental Engineering and Earth Sciences, Clemson University, Clemson, \\ SC 29634, USA \\ ${ }^{\mathrm{b}}$ Synterra, Greenville, SC 29601, USA \\ ${ }^{c}$ SiREM, Guelph, Ontario N1G 5G3, Canada \\ ${ }^{\mathrm{d}}$ Geosyntec Consultants International Inc., Toronto, Ontario, Canada
}

* Correspondence. dfreedm@clemson.edu 
1 ABSTRACT Chloroform (CF) and dichloromethane (DCM) are among the more commonly

2 identified chlorinated aliphatic compounds found in contaminated soil and groundwater. Complete

3 dechlorination of CF has been reported under anaerobic conditions by microbes that respire CF to

4 DCM and others that biodegrade DCM. The objectives of this study were to ascertain if a

5 commercially available bioaugmentation enrichment culture (KB-1 ${ }^{\circledR}$ Plus) uses an oxidative or

6 fermentative pathway for biodegradation of DCM; and to determine if the products from DCM

7 biodegradation can support organohalide respiration of CF to DCM in the absence of an exogenous

8 electron donor. In various treatments with the KB- $1{ }^{\circledR}$ Plus culture to which ${ }^{14} \mathrm{C}-\mathrm{CF}$ was added, the

9 predominant product was ${ }^{14} \mathrm{CO}_{2}$, indicating that oxidation is the predominant pathway for DCM.

10 Recovery of ${ }^{14} \mathrm{C}$-DCM when biodegradation was still in progress confirmed that CF first undergoes

11 reductive dechlorination to DCM. ${ }^{14} \mathrm{C}$-labeled organic acids, including acetate and propionate,

12 were also recovered, suggesting that synthesis of organic acids provides a sink for the electron

13 equivalents from oxidation of DCM. When the culture was washed to remove organic acids from

14 prior additions of exogenous electron donor and only CF and DCM were added, the culture

15 completely dechlorinated CF. The total amount of DCM added was not sufficient to provide the

16 electron equivalents needed to reduce CF to DCM. Thus, the additional reducing power came via

17 the DCM generated from CF reduction. Nevertheless, the rate of CF consumption was

18 considerably slower in comparison to treatments that received an exogenous electron donor. 
19 IMPORTANCE Chloroform (CF) and dichloromethane (DCM) are among the more commonly

20 identified chlorinated aliphatic compounds found in contaminated soil and groundwater. One way

21 to address this problem is to add microbes to the subsurface that can biodegrade these compounds.

22 While microbes are known that can accomplish this task, less is known about the pathways used

23 under anaerobic conditions. Some use an oxidative pathway, resulting mainly in carbon dioxide.

24 Others use a fermentative pathway, resulting in formation of organic acids. In this study, a

25 commercially available bioaugmentation enrichment culture (KB-1 ${ }^{\circledR}$ Plus) was evaluated using

26 carbon-14 labelled chloroform. The main product formed was carbon dioxide, indicating the use

27 of an oxidative pathway. The reducing power gained from oxidation was shown to support

28 reductive dechlorination of CF to DCM. The results demonstrate the potential to achieve full

29 dechlorination of CF and DCM to nonhazardous products that are difficult to identify in the field.

30

31 KEYWORDS Chloroform, dichloromethane, dehalorespiration, organohalide fermentation,

32 Dehalobacter, KB-1 ${ }^{\circledR}$ Plus 


\section{INTRODUCTION}

34 Considerable progress has been made in identifying the microbes and pathways involved in anaerobic biodegradation of chloroform (CF) and dichloromethane (DCM). For many decades, reductive dechlorination of CF to DCM was thought to be a cometabolic process (1). A key discovery by Grostern et al. (2) demonstrated the use of CF as a terminal electron acceptor during organohalide respiration by a Dehalobacter population. This built on the an earlier discovery that strain TCA1, a close relative to Dehalobacter restrictus, is able to respire using 1,1,1trichloroethane as its terminal electron acceptor (3). CF and 1,1,1-trichloroethane share the same

41 trichloro structure on a single carbon. The processes are also similar in that reductive

42 dechlorination stops before complete dechlorination is achieved, unlike what is possible with organohalide respiration of chlorinated ethenes (4). With CF, DCM is the main product, while respiration of 1,1,1-TCA, stops at chloroethane, both of which have potential environmental and toxicological impacts. Consequently, to achieve acceptable anaerobic remediation, further dechlorination is required to non-toxic end-products. for several decades $(5,6)$. Following development of highly enriched cultures that grow on DCM

49 (7), Leisinger and colleagues isolated Dehalobacterium formicoaceticum gen. nov. sp. nov. (8).

They subsequently proposed a modified version of the Wood-Ljungdahl pathway for fermentation

51 of DCM to a mixture of formate and acetate, involving a Co(I) corrinoid and tetrahydrofolate (9).

52 More than a decade passed before the next wave of research appeared, with a focus this time on 53 characterization of the RM consortium (10-12) and DCMF enrichment culture (13). DCM 54 degradation by the RM consortium, originally derived from pristine river sediment, was initially 55 attributed to Dehalobacter sp. $(14,15)$. The microbe in the RM consortium responsible for DCM 
degradation was subsequently identified as Candidatus Dichloromethanomonas elyunquensis.

57 The microbe enriched from organochlorine-contaminated groundwater near Botany Bay, Australia that was responsible for DCM degradation was initially identified as a Dehalobacter (16). Upon

59 further enrichment, the DCMF culture became dominated by a novel member of the Peptococcaceae family.

Various methods have been used to gain insight into the DCM biodegradation pathways used by the RM consortium and DCMF. Strain RM uses a mineralization pathway that oxidizes the carbon in DCM to $\mathrm{CO}_{2}$, with the electron equivalents (4 eeq/mol DCM) released as $\mathrm{H}_{2}$. The proposed pathway for DCMF is more similar to the fermentation steps used by Dehalobacterium formicoaceticum, with the principal products being acetate and formate. Based on free energy calculations, Chen et al. (17) predict that the mineralization pathway is predominant in environments with $\mathrm{H}_{2}$ levels below 100 parts per million by volume (ppmv). et al. (18) describe a microbial community that respires CF to DCM using emulsified vegetable oil or acetate as electron donors, followed by dehalofermentation of the DCM. Both processes were

71 attributed to Dehalobacter. Justica-Leon et al. (14) demonstrated reduction of CF to DCM

72 followed by consumption of DCM in microcosms bioaugmented with the CF-to-DCM 73 organohalide-respiring culture Dhb-CF and the DCM-degrading consortium RM. Electron donor

74 was supplied in the form of lactate and hydrogen. Neither of these studies explored the possibility 75 that complete dechlorination of CF is possible without addition of an electron donor, based on the 76 potential for fermentation products from DCM serving that purpose. A system similar to this has

77 been described for chlorobenzene. Liang et al. (19) combined a culture that respires chlorobenzene 78 to benzene with another culture that ferments the benzene to acetate and hydrogen, which serve as 
79 electron donors for the reduction process and therein remove the need for an exogenous electron

80 donor.

KB- $1^{\circledR}$ Plus is a commercially available bioaugmentation culture that is used to detoxify

82 contaminant plumes containing a variety of chlorinated aliphatic compounds. Prior studies have

83 demonstrated its effectiveness in dechlorinating chlorinated ethanes and CF $(2,20,21)$. The KB-

$84 \quad 1{ }^{\circledR}$ Plus formulation used in this study is a mixed culture that is maintained on CF and is known to

85 contain Dehalobacter spp., although the pathway used for biodegradation of DCM formed from

86 CF has not been reported. The objectives of this study were to ascertain if KB-1 ${ }^{\circledR}$ Plus uses an

87 oxidative or fermentative pathway for DCM, based on products formed from ${ }^{14} \mathrm{C}$-labeled $\mathrm{CF}$; and

88 to determine if the products from DCM biodegradation can support organohalide respiration of CF

89 to DCM in the absence of an exogenous electron donor.

90

91

92

93

94

95

96

97

98

99

100

101

\section{RESULTS}

Analysis of CF biodegradation using ${ }^{14} \boldsymbol{C}-\boldsymbol{C F}$. VOC results for one of the triplicates of treatment \#1 (without BES added) and \#3 (with BES added) are shown in Figure 1; results for other replicates and repeat experiments, as well as treatments $\# 2$ and $\# 4$, are shown in Supplementary Materials (Figures S1-S10). Treatment \#2 behaved similarly to treatment \#1 except that the incubation was stopped as soon as CF was consumed (Figures S4-S6). Only a minor amount of $\mathrm{CM}$ was detected in any of the bottles $(0.13 \mu \mathrm{mol}$ maximum, data points not shown), indicating that hydrogenolysis of DCM to $\mathrm{CM}$ was not an important part of the $\mathrm{CF}$ biodegradation pathway. In the absence of BES, the rate of CF and DCM biodegradation was approximately twice as fast. In both treatments, the maximum accumulation of DCM was approximately one third of the stoichiometric amount of $\mathrm{CF}$, indicating DCM fermentation occurred during hydrogenolysis of CF to DCM. Methanogenesis was inhibited by the presence of 
102 CF in treatment $\# 1$; once the CF was consumed, an average of $7 \mu \mathrm{mol}$ of methane accumulated.

103 By contrast, the presence of BES inhibited methanogenesis throughout the incubation. There was 104 only a minor decrease in CF in the medium A control (treatment \#4; Figure S10) and no 105 accumulation of DCM, CM, or methane. averages for 3 to 9 bottles in each treatment (Table 1). ${ }^{14} \mathrm{CO}_{2}$ was the predominant product in treatments with KB-1 ${ }^{\circledR}$ Plus present $\left(59-78 \%\right.$ of the initial ${ }^{14} \mathrm{C}-\mathrm{CF}$ added). ${ }^{14} \mathrm{C}-\mathrm{DCM}$ was significant only in treatment \#2 bottles that were sacrificed after CF was consumed but before all of the DCM was consumed. It is notable that there was also significant accumulation of ${ }^{14} \mathrm{CO}_{2}$ in these bottles, even before the DCM was completely consumed. ${ }^{14} \mathrm{C}-\mathrm{NSR}$, which includes organic 112 acids, comprised an average of $5.3 \%$ of the ${ }^{14} \mathrm{C}-\mathrm{CF}$ added, suggesting that the principal anaerobic biodegradation pathway for $\mathrm{CF}$ by $\mathrm{KB}-1^{\circledR}$ Plus is via mineralization, rather than direct 114 incorporation of carbon from DCM into organic acids. Samples of NSR from treatment \#3 were 115 separated by HPLC. The fraction with the highest level of ${ }^{14} \mathrm{C}$ activity $\left(62-69 \%\right.$ of the ${ }^{14} \mathrm{C}$ in the 116 NSR) had the same retention time as acetate; the next highest fraction $\left(6.4-13 \%\right.$ of the ${ }^{14} \mathrm{C}$ in the 117 NSR) eluted at the same time as propionate. Nearly complete recovery of ${ }^{14} \mathrm{C}-\mathrm{CF}(96 \%)$ and only minor amounts of ${ }^{14} \mathrm{C}-\mathrm{NSR}$ and ${ }^{14} \mathrm{CO}_{2}$ 119 in the medium control confirmed the high purity of the ${ }^{14} \mathrm{C}-\mathrm{CF}$ added and the lack of abiotic transformation (Figure 2). The amount of ${ }^{14} \mathrm{C}$ that was not accounted for was minor. The extent 121 of losses was a function of the incubation time, ranging from an average of $0.46 \%$ for the medium control ( 7 days of incubation) to $21 \%$ for treatment \#3 (23 days of incubation). 
125

126

127

128

129

130

131

132

133

134

135

136

137

138

139

140

141

142

143

144

145

146

147

no accumulation of $\mathrm{H}_{2}$ and methane formation that exceeded the level expected from DCM alone, i.e., $0.5 \mathrm{~mol} \mathrm{CH}_{4} / \mathrm{mol}$ DCM (Figure 3a). When the DCM dose was increased by an order of magnitude, a lag of approximately 30 days was followed by a rate of DCM consumption similar to the initial dose. The amount of methane produced during consumption of the second dose of DCM was closer to stoichiometric (i.e., $0.41 \mu \mathrm{mol} \mathrm{CH}_{4} / \mu \mathrm{mol} \mathrm{DCM}$ ), and was also accompanied by an increase in hydrogen. However, hydrogen represented a lower percentage of the electron flow versus methane (i.e., $6 \mu \mathrm{mol}$ of $\mathrm{H}_{2}$ accounts for $3 \mu \mathrm{mol}$ of DCM, out of $\sim 180 \mu \mathrm{mol}$ consumed). When BES was present along with the DCM in treatment \#6, the rate of DCM consumption was slowed and there was no accumulation of methane (Figure $3 b$ ). A transient decrease in the rate of DCM consumption occurred when hydrogen peaked at approximately $3 \mu \mathrm{mol}$ per bottle; as $\mathrm{H}_{2}$ levels declined, a higher rate of DCM consumption resumed. For treatments \#5, and \#6, CM remained below $0.5 \mu \mathrm{mol}$ per bottle.

The observation of hydrogen accumulation during consumption of DCM suggests that DCM may serve as the electron donor for reduction of CF to DCM. This was evaluated with treatment \#7, using the KB-1 ${ }^{\circledR}$ Plus culture as received. Consequently, acetate was initially present (as a product of lactate added as the electron donor). Repeat additions of $\sim 17 \mu \mathrm{mol}$ of CF and DCM per bottle were consumed within several days (Figure 4a), accompanied by accumulation of methane and no detectable hydrogen. As the dose of CF was increased around day 60, methane accumulation stopped, and DCM increased as CF was consumed. Once CF was below detection, the DCM was then completely consumed. This is best seen when the highest dose of CF was added on day 132. DCM increased to $75 \mu \mathrm{mol}$ per bottle on day 306 and then decreased below detection by day 336 , accompanied by an increase in $\mathrm{H}_{2}$ of $4.1 \mu \mathrm{mol}$ per bottle. The next three doses of CF and DCM followed a similar pattern. 

the amount possible (i.e., $0.5 \mu \mathrm{mol} \mathrm{CH}_{4}$ per $\mu \mathrm{mol} \mathrm{DCM}$ ) based on the peak level of DCM. A second dose of CF (16 $\mu \mathrm{mol} / \mathrm{bottle})$ on day 56 was accompanied by DCM $(3.6 \mu \mathrm{mol} / \mathrm{bottle})$, and evaluated for organic acids at the start and end of the incubation. Although small peaks were inoculum. Lactate, propionate, and citrate were below detection in all of the bottles.

\section{DISCUSSION}

Based on the ${ }^{14} \mathrm{C}$ results (Fig. 2), a proposed pathway for anaerobic biodegradation of CF

by the KB- $1{ }^{\circledR}$ Plus culture is shown in Figure $5 .{ }^{14} \mathrm{CO}_{2}$ was the predominant product for the live consumed but DCM was still present. As expected, the DCM was ${ }^{14} \mathrm{C}$-labelled, confirming that culture uses an oxidative pathway for anaerobic biodegradation of DCM, similar to the RM consortium (17). The medium used for cultivation contains $20 \mathrm{mM}$ of bicarbonate. If all 17.2 
$171 \mathrm{CO}_{2}$ would be $0.17 \mathrm{mM}$. Because this is orders of magnitude lower than the bicarbonate level in

172 the medium, the ${ }^{14} \mathrm{CO}_{2}$ formed was diluted by a much larger pool of bicarbonate, which would

173 predominate over $\mathrm{CO}_{2}$ in the circumneutral medium.

174 Oxidation of DCM to $\mathrm{CO}_{2}$ yields 4 electron equivalents (eeq) per mol, or 2 mol $\mathrm{H}_{2}$ (Fig.

175 5). One $\mathrm{mol} \mathrm{H}_{2}$ is then available to accomplish reduction of $1 \mathrm{~mol} \mathrm{CF}$ to DCM. That leaves a net

176 of $1 \mathrm{~mol} \mathrm{H}_{2}$ per mol of CF. With treatments \#5-8, $\mathrm{H}_{2}$ accumulated, but even the highest levels

177 observed were never close to the stoichiometric level (Figs. 3 and 4). That indicated there must

178 be a sink for this reducing power. Formation of organic acids is one possibility. With treatments

$179 \# 1-3$, approximately $3-5 \%$ of the ${ }^{14} \mathrm{C}$ was recovered as nonstrippable residue (NSR), and HPLC

analysis of NSR from treatment \#3 indicated the main components were (presumptively) acetate

181 and propionate. However, because the ${ }^{14} \mathrm{CO}_{2}$ generated from ${ }^{14} \mathrm{C}-\mathrm{CF}$ was diluted in bicarbonate

182 present in the medium, most of the organic acids formed would likely be unlabeled. This would

183 make them difficult to quantify in treatments \#1-3, because of the background level of organic 184 acids present in the KB-1 ${ }^{\circledR}$ Plus culture as received from SiREM.

One of the objectives with treatment \#8 was to improve the potential to quantify the amount

186 of organic acids that can form when $\mathrm{CF}$ is biodegraded by the KB-1 ${ }^{\circledR}$ Plus culture. The culture in

187 these bottles was washed to remove the background level of organic acids prior to addition of CF

188 and lesser amounts of DCM (Fig. 4). If all of the $19.8 \mu \mathrm{mol}$ of CF and $4.0 \mu \mathrm{mol}$ of DCM were

189 oxidized to $\mathrm{CO}_{2}$, then 55.6 eeq of reducing power would be available to form organic acids (i.e.,

$19019.8 \mu \mathrm{mol} \mathrm{CF} * 2 \mu \mathrm{eeq} / \mu \mathrm{mol}+4.0 \mu \mathrm{mol} \mathrm{DCM} * 4 \mu \mathrm{eeq} / \mu \mathrm{mol})$. Conversion of 55.6 eeq per bottle

191 (with $44 \mathrm{~mL} / \mathrm{bottle})$ to acetate $(8 \mu \mathrm{eeq} / \mu \mathrm{mol})$ would yield a maximum concentration of $158 \mu \mathrm{M}$.

192 If the reducing power was used to make formate $(6 \mu \mathrm{eeq} / \mu \mathrm{mol})$ the outcome would be $211 \mu \mathrm{M}$.

193 These concentrations are well above the detection limit. Nevertheless, at the end of the incubation 
194 period, there was no statistically significant accumulation of organic acids in comparison to the 195 controls. The extended incubation period (431-477 days) may have complicated closing the mass 196 balance on electron equivalents. If the KB-1 ${ }^{\circledR}$ Plus culture can be acclimated to more rapid 197 consumption of CF and DCM in the absence of an exogenous substrate, it may become more 198 feasible to close the balance. Another approach would be to substantially lower the bicarbonate 199 concentration in the medium, so that more of the ${ }^{14} \mathrm{CO}_{2}$ could be used to form organic acids.

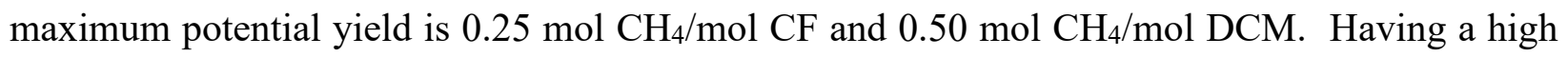
background level of organic acids in treatments $\# 1-3$ and \#5-7 limited the option to quantify methane formation that is attributable only to CF and DCM (e.g., Fig. 1a, 3a, and 4a). With removal of the residual organic acids from treatment \#8, the maximum possible yield of methane from the CF and DCM consumed was $7.0 \mu \mathrm{mol}$ per bottle. The observed increase in the treatment \#8 bottles was less than $0.10 \mu \mathrm{mol}$ per bottle, well below what is needed to close the eeq balance. Furthermore, with treatments in which methanogenesis was inhibited (i.e., \#3 and \#6 with BES added, and \#7 when the CF concentration was increased above $\sim 22 \mu \mathrm{mol} /$ bottle, or an aqueous phase concentration of $\sim 24 \mathrm{mg} / \mathrm{L}$ ), CF was still consumed to completion, so that methanogenesis was eliminated as a sink for the eeq from oxidation of DCM.

$213(5,17)$. This is made possible by the large Gibbs free energy change derived from breaking the 214 carbon chlorine bonds. In spite of the thermodynamic favorability of DCM oxidation even at 1 215 atm $\mathrm{H}_{2}$, the $\mathrm{RM}$ consortium requires the presence of $\mathrm{H}_{2}$-consuming partners. It is evident from 216 this study that lower levels of $\mathrm{H}_{2}$ also favor higher rates of CF consumption by the KB-1 ${ }^{\circledR}$ Plus 
217 culture (Fig. 3). Inhibition of methanogenesis (with either BES or higher levels of CF) led to

218 slower rates of CF and DCM degradation (Fig. 1, 3, and 4). Methanogenesis contributes to keeping

219 the partial pressure of hydrogen at a low level. Chen et al. (17) speculated that $\mathrm{H}_{2}$ inhibition may

220 be related to negative impacts on $\mathrm{H}_{2}$-evolving hydrogenases present in the $\mathrm{RM}$ consortium.

For treatments \#1-3 and \#5-7, accumulation of DCM was never stoichiometric with respect

222 to the amount of CF added, indicating that DCM biodegradation was underway as CF was reduced

223 to DCM. Nevertheless, DCM typically increased as CF was consumed and a decreasing trend for

224 DCM did not occur until CF was mostly or completely consumed (Fig. 1, 4a). This is consistent

225 with a previous report that CF inhibits DCM biodegradation, even at CF concentrations as low as

$2265 \mathrm{mg} / \mathrm{L}(15)$. The fact that repeat additions of CF and DCM were consumed indicates that this

227 inhibition is reversible with the KB-1 ${ }^{\circledR}$ Plus culture.

possible without addition of an exogenous electron donor. The source of electron donor for CF reduction to DCM is the oxidation of DCM. The culture used for treatment \#8 was washed to remove the accumulated organic acids, yet biodegradation of CF proceeded. The process was 232 "jump started" by adding DCM along with both doses of CF (Fig. 4). Nevertheless, the total amount of DCM added $(3.96 \mu \mathrm{mol} /$ bottle $)$ provided $15.8 \mu$ eeq of reducing power, while the total 234 amount of CF consumed $(19.75 \mu \mathrm{mol} /$ bottle) required $39.5 \mu \mathrm{eeq}$ of reducing power. Thus, the 235 addition reducing power needed must have been made available by the DCM generated from CF 236 reduction. While sustaining CF dechlorination without an input of electron donor is of interest, 237 the rate of CF consumption was considerably slower in comparison to treatments that received 238 electron donor in the form of lactate (Fig. 1). For this reason, it is still advisable to use an electron 239 donor in the field to accelerate the rate of CF reduction to DCM. 

useful for bioremediation because the products formed are not discernable from the background, so it is not possible to use product accumulation as evidence of in situ biodegradation. Quantifying opportunities to document in situ bioremediation of CF.

\section{MATERIALS AND METHODS}

Chemicals and enrichment culture. CF (99.7\%) was obtained from Shelton Scientific. lactate syrup (containing 58.8-61.2\% sodium lactate; specific gravity $=1.31$ ) from Spectrum

255 cocktail (LSC) (50\%) from Fisher Scientific; and 2-bromoethanesulfonic acid (BES; sodium salt, 98\%) and sodium propionate (99\%) from Sigma Aldrich. All other chemicals were reagent grade.

258 composition is described previously (22). The KB-1 ${ }^{\circledR}$ Plus enrichment culture and lactate-free

259 anaerobic mineral medium B were provided by SiREM and shipped on ice to Clemson University 260 via an overnight carrier. Upon receipt, the culture and the medium were placed in an anaerobic chamber and allowed to warm to room temperature. 
Experimental design. The experimental design includes the use of ${ }^{14} \mathrm{C}-\mathrm{CF}$, the presence

263 of various electron donors, and use of BES to inhibit methanogenesis (Table 1). One set of

264 experiments (treatments \#1-4) was performed with ${ }^{14} \mathrm{C}-\mathrm{CF}$ for the purpose of determining the ${ }^{14} \mathrm{C}$

265 products that formed. These experiments utilized KB-1 ${ }^{\circledR}$ Plus (CF formulation) as received and

266 lactate as the primary electron donor. A second set of experiments evaluated use of DCM as an

267 electron donor in support of hydrogenolysis of CF to DCM. Treatments \#5 and 6 evaluated DCM

268 as the sole substrate added; treatments \#7-9 included both CF and DCM. Treatment \#10 served as

269 a control for product formation from the inoculum used in treatment \#8. Acetate was present as a

270 fermentation product from lactate except when it was removed by washing the culture (treatments

$271 \quad \# 8$ and 9; described below).

272 Treatments \#1-7 were prepared in $160 \mathrm{~mL}$ serum bottles with $100 \mathrm{~mL}$ of culture or medium;

273 treatments $\# 8$ and 9 were prepared in $70 \mathrm{~mL}$ serum bottles with $44 \mathrm{~mL}$ of washed culture or

274 medium; treatment \#9 was prepared in $12 \mathrm{~mL}$ serum bottles with $3 \mathrm{~mL}$ of washed culture. The

275 serum bottles were capped with Teflon-faced grey butyl rubber septa; the bottles and septa were

276 autoclaved prior to use. After dispensing the culture in an anaerobic chamber, the serum bottles

277 were removed, the headspace was sparged with $\mathrm{N}_{2} / \mathrm{CO}_{2}(70 \% / 30 \%)$ to remove hydrogen that was

278 present in the atmosphere of the anaerobic chamber, and the bottles were recapped. CF and DCM

279 were added as water saturated solutions ( $\sim 67$ and $235 \mathrm{mM}$, respectively). After adding $\mathrm{CF}$ and/or

280 DCM, the serum bottles were mixed on a shaker table (100 rpm for $1 \mathrm{~h}$ ) to allow the CF and/or

281 DCM to equilibrate between the headspace and liquid, sampled for analysis of the volatile

282 compounds, and returned to the anaerobic chamber where they were incubated quiescently until

283 the next sampling event. Incubation was at room temperature $\left(\sim 22-24{ }^{\circ} \mathrm{C}\right)$. 
Fate of $\mathbf{C F}$ using ${ }^{14} \mathbf{C}-\mathbf{C F}$. The products from anaerobic degradation of CF were assessed

285

286

287

288

289

290

291

292

293

294

295

296

297

298

299

300

301

302

303

304

305

306

using ${ }^{14} \mathrm{C}-\mathrm{CF}$. Treatment \#1 (Table 1) was performed in triplicate on three occasions (9 bottles

total), with lactate as the electron donor. Degradation was allowed to continue until CF and DCM

were below detection. For two of the sets of triplicates ( 6 bottles), the ability of the culture to

consume unlabeled CF was confirmed prior to adding the ${ }^{14} \mathrm{C}-\mathrm{CF}$. Treatment \#2 was prepared

identically but incubation was stopped when at least one third of the maximum possible

stoichiometric level of DCM remained. Treatment \#3 was the same as \#1 except that BES (55

$\mathrm{mM}$ ) was added to inhibit methanogenesis (23); two sets of triplicates were evaluated (6 bottles

total). Treatment \#4 served as a control to assess abiotic loss of ${ }^{14} \mathrm{C}-\mathrm{CF}$ with only medium A

present.

CF-saturated water $(0.25 \mathrm{~mL} /$ bottle $)$ and a stock solution of sodium lactate $(24 \mu \mathrm{L} /$ bottle

of a stock solution containing $650 \mathrm{~g} / \mathrm{L}$ of $60 \%$ sodium lactate syrup) were added. The ratio of electron equivalents of lactate added to equivalents needed to reduce CF to DCM was approximately 20. Next, ${ }^{14} \mathrm{C}-\mathrm{CF}$ was added using an aqueous stock solution (see below).

Headspace and liquid samples were used to establish the initial amount of ${ }^{14} \mathrm{C}-\mathrm{CF}$ present. Thereafter, the bottles were incubated quiescently. Headspace samples were removed periodically to determine the total levels of CF, DCM, CM, and methane by gas chromatography (see below). For treatment \#2, triplicate bottles were sacrificed to determine the distribution of ${ }^{14} \mathrm{C}$ (see below) when DCM degradation was incomplete. Treatments \#1 and \#3 were incubated until CF and DCM were no longer detectable, at which point these and the medium controls (treatment \#4) were sacrificed to determine the distribution of ${ }^{14} \mathrm{C}$.

The presence of acetate at the start of the incubation was a consequence of prior additions of lactate to the culture. Acetate is a product of lactate fermentation. The initial concentration of 
307

308

309

310

311

312

313

314

315

316

317

318

319

320

321

322

323

324

325

326

327

328

329

acetate was $\sim 27 \mathrm{mM}$. Prior evaluation of the KB-1 ${ }^{\circledR}$ culture (from which KB-1 ${ }^{\circledR}$ Plus was developed) indicates that acetate does not serve as an electron donor for reductive dechlorination (24).

Evaluation of CF and DCM as sole substrates. Treatments \#5-10 explored the potential for $\mathrm{CF}$ and DCM to serve as sole substates for the $\mathrm{KB}-1{ }^{\circledR}$ Plus culture; ${ }^{14} \mathrm{C}-\mathrm{CF}$ was not used. Treatment \#5 received DCM as the source of carbon and energy. After an initial dose of 16.5 $\mu \mathrm{mol}$ DCM per bottle was consumed, a second dose of $165 \mu \mathrm{mol}$ was added. Treatment \#6 was identical to \#5 except that BES was added, along with one dose of DCM (16.5 $\mu$ mol was consumed). Methane and hydrogen were quantified along with DCM.

Treatment \#7 received CF and DCM. After the initial amount of CF was consumed (1.68 $\mu$ mol per bottle), increasing amounts were added (up to $98 \mu \mathrm{mol}$ ), while the amount of DCM added remained constant $(\sim 15 \mu \mathrm{mol}$ per bottle). Prior to setting up the bottles for treatments \#5-7, DCM $(165 \mu \mathrm{M})$ was added to one liter of the $\mathrm{KB}-1^{\circledR}$ Plus culture to confirm the activity of the culture. As with treatment $\# 1,2,3,5$, and 6 , acetate was present at the start of the incubation as a product of prior addition of lactate as the electron donor.

Treatment \#8 differed from \#7 as follows: The source of inoculum was one of the serum bottles from treatment \#7 (100 mL); the culture was washed to remove residual organic acids; the initial dose of CF was lower (4.0 $\mu \mathrm{mol}$ per bottle) and a subsequent CF dose added was higher (16 $\mu \mathrm{mol}$ per bottle); and three lower doses of DCM were added $(0.33,0.16$, and $3.47 \mu \mathrm{mol}$ per bottle on days 0,14 , and 56 , respectively). Washing was accomplished by centrifugation (1880-g, 30 min), decanting, and resuspending in $145 \mathrm{~mL}$ of medium B. Acetate was below detection in the resuspended culture. After washing, the color of resazurin in the medium turned slightly pink, indicative of a redox level above $-110 \mathrm{mV}$. To restore a lower redox level, $8.8 \mu \mathrm{mol}$ of $\mathrm{Na}_{2} \mathrm{~S}$ was 
added $\left(100 \mu \mathrm{L}\right.$ of a stock solution of $\left.88 \mathrm{mM} \mathrm{Na} 2 \mathrm{~S} \cdot 9 \mathrm{H}_{2} \mathrm{O}\right)$. The washed culture was dispensed to triplicate $70 \mathrm{~mL}$ serum bottles, each with $44 \mathrm{~mL}$ of culture.

Two controls accompanied treatment \#8. Treatment \#9 consisted of the washed and resuspended KB- $1{ }^{\circledR}$ Plus culture, with no CF or DCM added; $3.0 \mathrm{~mL}$ was dispensed to triplicate $12 \mathrm{~mL}$ serum bottles. Treatment \#10 consisted of only medium B in $70 \mathrm{~mL}$ serum bottles, with similar initial doses of $\mathrm{CF}$ and DCM. At the end of the incubation period, aliquots from treatments \#8-10 were analyzed for organic acids.

The $\mathrm{pH}$ in treatments \#7 and 8 was monitored periodically; when the $\mathrm{pH}$ fell below 6.5 in treatment \#7, sodium bicarbonate $(100 \mu \mathrm{L}$ of a stock solution of $10 \mathrm{~g} / \mathrm{L}$ sodium bicarbonate $)$ was added (day 398) to restore circumneutral conditions. The $\mathrm{pH}$ remained circumneutral in treatment \#8.

Analytical methods. ${ }^{14} \mathrm{C}$-CF was purified as previously described for ${ }^{14} \mathrm{C}$-labeled trichloroethene (25). Briefly, the ${ }^{14} \mathrm{C}-\mathrm{CF}$ stock solution $(100 \mu \mathrm{L})$ was injected onto a gas chromatograph and passed through the same packed column used to analyze the volatile organic compounds (see below). The outlet of the column was connected to stainless steel tubing that exited the GC oven and terminated with a needle, which was inserted into the microcosm headspace during the interval when $\mathrm{CF}$ eluted ( 6.5-7.0 min). $\mathrm{N}_{2}$ was used as the carrier gas (28.5 $\mathrm{mL} / \mathrm{min}$ at $150{ }^{\circ} \mathrm{C}$ ). Preliminary experiments with water controls confirmed that the only volatile compound present following this procedure was $\mathrm{CF}$.

The total initial amount of ${ }^{14} \mathrm{C}$ present in the serum bottles was quantified by counting samples of the headspace $(0.5 \mathrm{~mL})$ and liquid $(1 \mathrm{~mL})$ in $15 \mathrm{~mL}$ of liquid scintillation cocktail. The procedure to determine the distribution of ${ }^{14} \mathrm{C}$-labeled materials remaining in a microcosm at the time it was sacrificed is described by Darlington et al. (26). Volatile ${ }^{14} \mathrm{C}$-labeled biodegradation 
353 products (primarily DCM) were analyzed with a GC-combustion technique. A 0.5-mL headspace

354 sample from the microcosms was injected onto the GC column and the separated compounds were 355 routed to a catalytic combustion tube (containing $\mathrm{CuO}$ ) at $800^{\circ} \mathrm{C}$, where compounds were oxidized 356 to $\mathrm{CO}_{2}$. Each fraction was then trapped in $3 \mathrm{~mL}$ of $0.5 \mathrm{M} \mathrm{NaOH}$, which was then mixed with 15 $357 \mathrm{~mL}$ of LSC. The efficiency of the combustion technique (i.e., $\Sigma\left({ }^{14} \mathrm{C}\right.$ in the fractions) divided by 358 the ${ }^{14} \mathrm{C}$ in a headspace sample) averaged $101 \pm 20 \%$. To measure ${ }^{14} \mathrm{CO}_{2}$ and ${ }^{14} \mathrm{C}$-labeled nonvolatile products, liquid samples from the serum 360 bottles were acidified and then sparged with $\mathrm{N}_{2}$, which was passed through an alkaline solution. 361 The compounds remaining in the acidified sample were classified as nonstrippable residue (NSR); 362 the activity in the alkaline solution was presumed to be ${ }^{14} \mathrm{CO}_{2}(26)$. Formation of ${ }^{14} \mathrm{CO}_{2}$ was 363 confirmed by a precipitation test using barium hydroxide (26). The distribution of ${ }^{14} \mathrm{C}$ includes an 364 "unaccounted for" category, calculated as the total ${ }^{14} \mathrm{C}$ remaining in a bottle minus the identified 365 products $\left({ }^{14} \mathrm{C}-\mathrm{CF},{ }^{14} \mathrm{C}-\mathrm{DCM},{ }^{14} \mathrm{CO}_{2}\right.$ and $\left.{ }^{14} \mathrm{C}-\mathrm{NSR}\right)$, and losses. Losses were calculated as the ${ }^{14} \mathrm{C}$ 366 added at time zero minus the total ${ }^{14} \mathrm{C}$ remaining at the end of the incubation period. The percentage of ${ }^{14} \mathrm{C}-\mathrm{NSR}$ in bottles for treatment \#3 was sufficiently high $(\sim 5 \%$ of the 368 total ${ }^{14} \mathrm{C}$ added) to warrant further characterization by fractionation on the same high performance 369 liquid chromatograph (HPLC) used to quantify organic acids (see below). A filtered $(0.20 \mu \mathrm{m})$ 370 aliquot of NSR $(100 \mu \mathrm{L})$ was injected onto the HPLC and fractions were captured in LSC as they 371 eluted off the column, in intervals of $1.70 \mathrm{~min}$ (except for the intervals corresponding to formate 372 and acetate, which were narrowed to 1.05 and $1.15 \mathrm{~min}$, respectively). This was repeated with two 373 more injections per bottle, so that the total volume of eluant captured per LSC vial was 1.9-3.1 mL. 374 The ${ }^{14} \mathrm{C}$ activity in each fraction was then counted (see below). 
A PerkinElmer Tri-Carb ${ }^{\circledR} 2910$ TR liquid scintillation counter was used to quantify ${ }^{14} \mathrm{C}$

376

377

378

379

380

381

382

383

384

385

386

387

388

389

390

391

392

\section{Supplemental Material}

394 Figures S1-S3: replicates for Figure 1a;

395 Figures S4-S6: VOC results for treatment \#2;

396 Figures S7-S9: replicates for Figure 1b; 
397 Figure S10: VOC results for treatment \#4;

398 Figure S11: replicates for Figure 3a;

399 Figure S12: replicates for Figure 3b;

$400 \quad$ Figure S13: replicates for Figure 4a;

$401 \quad$ Figure S14: replicates for Figure 4b; and

402 Figure S15: VOC results for treatment \#10.

\section{REFERENCES}

404 1. Cappelletti M, Frascari D, Zannoni D, Fedi S. 2012. Microbial degradation of

405 chloroform. Applied Microbiology and Biotechnology 96:1395-1409.

406 2. Grostern A, Duhamel M, Dworatzek S, Edwards EA. 2010. Chloroform respiration to 407 dichloromethane by a Dehalobacter population. Environmental Microbiology 12:1053$408 \quad 1060$.

409 3. Sun B, Griffin BM, Ayala-del-Rio HL, Hashsham SA, Tiedje JM. 2002. Microbial 410 dehalorespiration with 1,1,1-trichloroethane. Science 298:1023-1025.

411 4. Adrian L, Löffler FE. 2016. Organohalide-Respiring Bacteria. Springer, Berlin.

412 5. Freedman DL, Gossett JM. 1991. Biodegradation of dichloromethane and its utilization 413 as a growth substrate under methanogenic conditions. Appl Environ Microbiol 57:2847-

414 2857.

415 6. Stromeyer SA, Winkelbauer W, Kohler H, Cook AM, Leisinger T. 1991.

416 Dichloromethane utilized by an anaerobic mixed culture: acetogenesis and 417 methanogenesis. Biodegradation 2:129-137. 
418 7. Mägli A, Rainey FA, Leisinger T. 1995. Acetogenesis from dichloromethane by a twocomponent mixed culture comprising a novel bacterium. Appl Environ Microbiol

420 61:2943-2949.

421 8. Mägli A, Wendt M, Leisinger T. 1996. Isolation and characterization of Dehalobacterium formicoaceticum gen. nov. sp. nov., a strictly anaerobic bacterium utilizing dichloromethane as source of carbon and energy. Archives of Microbiology 166:101-108.

424 9. Mägli A, Messmer M, Leisinger T. 1998. Metabolism of dichloromethane by the strict anaerobe Dehalobacterium formicoaceticum. Appl Environ Microbiol 64:646-650.

10. Kleindienst S, Chourey K, Chen G, Murdoch RW, Higgins SA, Iyer R, Campagna SR, Mack EE, Seger ES, Hettich RL, Löffler FE, Parales RE. 2019. Proteogenomics reveals novel reductive dehalogenases and methyltransferases expressed during anaerobic dichloromethane metabolism. Appl Environ Microbiol 85:e02768-18.

430 11. Kleindienst S, Higgins SA, Tsementzi D, Chen G, Konstantinidis KT, Mack EE, Löffler FE. 2017. 'Candidatus Dichloromethanomonas elyunquensis' gen. nov., sp. nov., a dichloromethane-degrading anaerobe of the Peptococcaceae family. Systematic Appl

434 12. Kleindienst S, Higgins SA, Tsementzi D, Konstantinidis KT, Mack EE, Löffler FE. 2016. 435 Draft genome sequence of a strictly anaerobic dichloromethane-degrading bacterium. Genome Announcements 4:e00037-16.

437 13. Holland SI, Edwards RJ, Ertan H, Wong YK, Russell TL, Deshpande NP, Manefield MJ, Lee M. 2019. Whole genome sequencing of a novel, dichloromethane-fermenting Peptococcaceae from an enrichment culture. PeerJ 7:e7775. 
440 14. Justicia-Leon SD, Higgins S, Mack EE, Griffiths DR, Tang S, Edwards EA, Löffler FE.

441

442

443

444

445

446

447

448

449

450

451

452

453

454

455

456

457

458

459

460

461

462

2014. Bioaugmentation with distinct Dehalobacter strains achieves chloroform detoxification in microcosms. Environ Sci Technol.

15. Justicia-Leon SD, Ritalahti KM, Mack EE, Löffler FE. 2012. Dichloromethane fermentation by a Dehalobacter sp. in an enrichment culture derived from pristine river sediment. Appl Environ Microbiol 78:1288-1291.

16. Lee M, Low A, Zemb O, Koenig J, Michaelsen A, Manefield M. 2012. Complete chloroform dechlorination by organochlorine respiration and fermentation. Environ Microbiol 14:883-894.

17. Chen G, Fisch AR, Gibson CM, Erin Mack E, Seger ES, Campagna SR, Löffler FE. 2020. Mineralization versus fermentation: evidence for two distinct anaerobic bacterial degradation pathways for dichloromethane. ISME Journal 14:959-970.

18. Lee M, Low A, Zemb O, Koenig J, Michaelsen A, Manefield M. 2012. Complete chloroform dechlorination by organochlorine respiration and fermentation. Environmental Microbiology 14:883-894.

19. Liang X, Devine CE, Nelson J, Sherwood Lollar B, Zinder S, Edwards EA. 2013. Anaerobic conversion of chlorobenzene and benzene to $\mathrm{CH}_{4}$ and $\mathrm{CO}_{2}$ in bioaugmented microcosms. Environ Sci Technol 47:2378-2385.

20. Grostern A, Edwards EA. 2006. Growth of Dehalobacter and Dehalococcoides spp. during degradation of chlorinated ethanes. Appl Environ Microbiol 72:428-436.

21. Jones EJP, Voytek MA, Lorah MM, Kirshtein JD. 2006. Characterization of a microbial consortium capable of rapid and simultaneous dechlorination of 1,1,2,2-tetrachloroethane and chlorinated ethane and ethene intermediates. Bioremediation Journal 10:153-168. 
463 22. Freedman DL, Gossett JM. 1989. Biological reductive dechlorination of tetrachloroethylene and trichloroethylene to ethylene under methanogenic conditions. Appl Environ Microbiol 55:2144-2151.

466 23. Gunsalus RP, Romesser JA, Wolfe RS. 1978. Preparation of coenzyme M analogues and their activity in the Methyl Coenzyme M Reductase system of Methanobacterium

469 24. Duhamel M, Wehra SD, Yua L, Rizvia H, Seepersada D, Dworatzeka S, Cox EE, Edwards EA. 2002. Comparison of anaerobic dechlorinating enrichment cultures maintained on tetrachloroethene, trichloroethene, cis-dichloroethene and vinyl chloride.

473 25. Mills IV JC, Wilson JT, Wilson BH, Wiedemeier TH, Freedman DL. 2018. Monitoring \& Rem 38:57-67.

476 26. Darlington R, Lehmicke L, Andrachek RG, Freedman DL. 2008. Biotic and abiotic anaerobic transformations of trichloroethene and cis-1,2-dichloroethene in fractured sandstone. Environ Sci Technol 42:4323-4330.

479 27. Gossett JM. 1987. Measurement of Henry's Law constants for $\mathrm{C}_{1}$ and $\mathrm{C}_{2}$ chlorinated $480 \quad$ hydrocarbons. Environ Sci Technol 21:202-208.

481 28. Elango V, Kurtz Jr. HD, Anderson C, Freedman DL. 2011. Use of $\gamma$ hexachlorocyclohexane as a terminal electron acceptor by an anaerobic enrichment culture. J Haz Mat 197:204-210.

484 29. Chen G, Shouakar-Stash O, Phillips E, Justicia-Leon SD, Gilevska T, Sherwood Lollar B, 
distinct anaerobic dichloromethane degradation pathways in two members of Peptococcaceae. Environ Sci Technol 52:8607-8616.

488 30. Trueba-Santiso A, Parladé E, Rosell M, Lliros M, Mortan SH, Martínez-Alonso M, Gaju

489 N, Martín-González L, Vicent T, Marco-Urrea E. 2017. Molecular and carbon isotopic characterization of an anaerobic stable enrichment culture containing Dehalobacterium sp. during dichloromethane fermentation. Sci Total Environ 581-582:640-648. 
Table 1. Experimental design.

\begin{tabular}{|c|c|c|c|c|c|c|c|c|c|}
\hline $\begin{array}{c}\text { Treatment } \\
\#\end{array}$ & Contents & $\mathrm{n}^{a}$ & $\begin{array}{l}{ }^{14} \mathrm{C}-\mathrm{CF} \\
(\mu \mathrm{Ci})^{b}\end{array}$ & $\begin{array}{c}\mathrm{CF} \\
(\mu \mathrm{mol})^{b}\end{array}$ & $\begin{array}{c}\text { DCM } \\
(\mu \mathrm{mol})^{b}\end{array}$ & $\begin{array}{l}\text { DCM } \\
\text { status }^{c}\end{array}$ & Lactate $^{d}$ & Acetate $^{e}$ & BES \\
\hline 1 & KB- $1^{\circ}$ Plus & $9(6)$ & 0.36 & 17.2 & 0 & $\mathrm{C}$ & Yes & Yes & No \\
\hline 2 & KB-1 $1^{\circ}$ Plus & $9(6)$ & 0.34 & 17.2 & 0 & $\mathrm{NC}$ & Yes & Yes & No \\
\hline 3 & KB-1 $1^{\bullet}$ Plus & $6(6)$ & 0.37 & 17.2 & 0 & $\mathrm{C}$ & Yes & Yes & Yes \\
\hline 4 & Medium $\mathrm{A}^{f}$ & 3 & 0.38 & 15.7 & 0 & - & - & - & - \\
\hline 5 & KB-1 $1^{\circ}$ Plus & 3 & 0 & 0 & 16.5 & $\mathrm{C}$ & No & Yes & No \\
\hline 6 & KB-1 $1^{\circ}$ Plus & 3 & 0 & 0 & 16.5 & $\mathrm{C}$ & No & Yes & Yes \\
\hline 7 & KB-1 ${ }^{\bullet}$ Plus & 3 & 0 & 1.68 & 16.5 & $\mathrm{C}$ & No & Yes & No \\
\hline 8 & KB- $1^{\oplus} \mathrm{Plus} / \mathrm{w}^{g}$ & 3 & 0 & 4.02 & 0.47 & $\mathrm{C}$ & No & No & No \\
\hline 9 & KB $-1^{\circ}$ Plus $/ w$ & 3 & 0 & 0 & 0 & - & No & No & No \\
\hline 10 & Medium $\mathrm{B}^{f}$ & 3 & 0 & 4.02 & 0.94 & - & - & - & - \\
\hline
\end{tabular}

${ }^{a}$ Number of microcosms. Values in parenthesis represent number of bottles that received a dose of unlabeled $\mathrm{CF}$ prior to adding ${ }^{14} \mathrm{C}-\mathrm{CF}$.

${ }^{b}$ Initial amount added.

${ }^{c} \mathrm{C}=$ complete $\mathrm{DCM}$ degradation; $\mathrm{NC}=\mathrm{DCM}$ degradation was not complete.

${ }^{d}$ Lactate added as the electron donor.

$e$ Acetate present at the start of the incubation as a byproduct from prior addition of lactate.

$f$ Media A and B are described in Materials and Methods.

$g / \mathrm{w}$ indicates the culture was washed prior to incubation. 

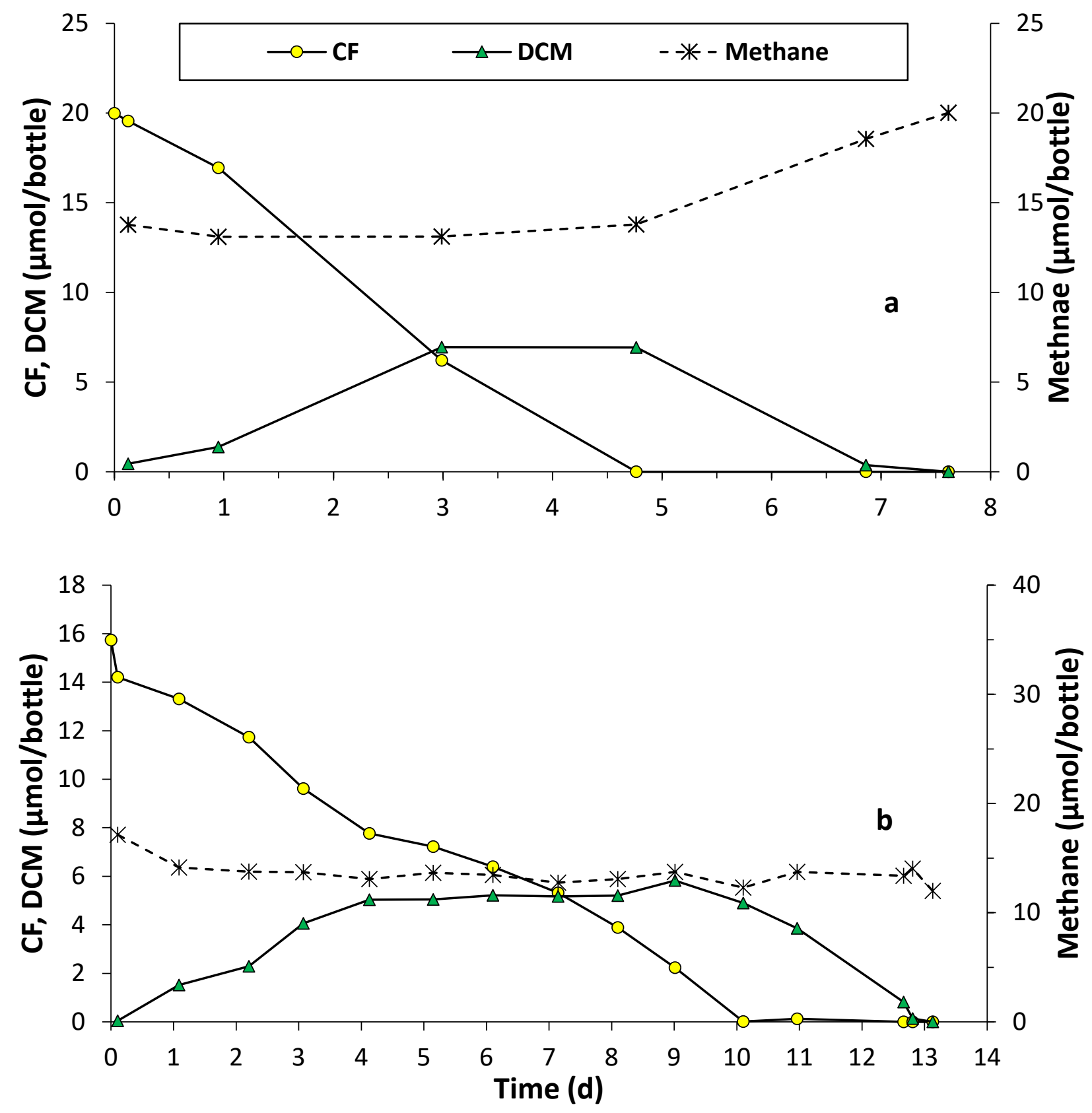

Figure 1. Representative results for biodegradation of CF by the KB-1 Plus ${ }^{\circledR}$ culture with the addition of lactate (electron donor) a) treatment \#1, without BES added; and b) treatment \#3, with BES added. One dose of lactate was added to all bottles at time zero. Results for single bottles are shown; replicates behaved similarly (Supplementary Material). Results for treatments \#2 and \#4 are provided in the Supplementary Material. 


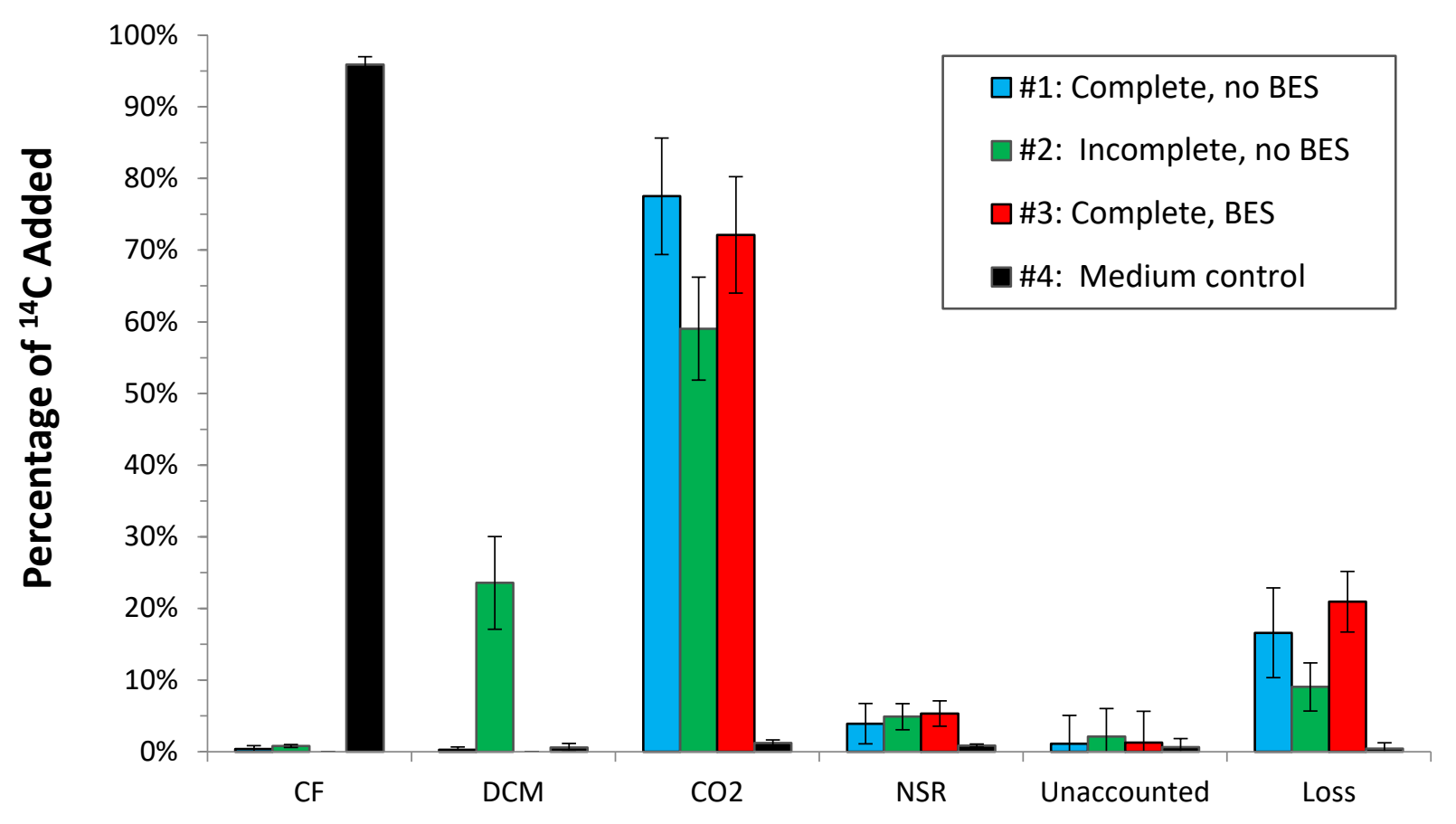

Figure 2. Percentage of ${ }^{14} \mathrm{C}$ from ${ }^{14} \mathrm{C}-\mathrm{CF}$ during biodegradation by KB-1 ${ }^{\oplus}$ Plus in treatments $\# 1-4$. Error bars represent the standard deviation; the number of replicates for each treatment are shown in Table 1. "Complete" means all of the CF and DCM were consumed. "Incomplete" means the CF was consumed but not all of the DCM was consumed. BES is 2bromoethanesulfonate, an inhibitor of methanogenesis. Medium A was used for treatment \#4. 

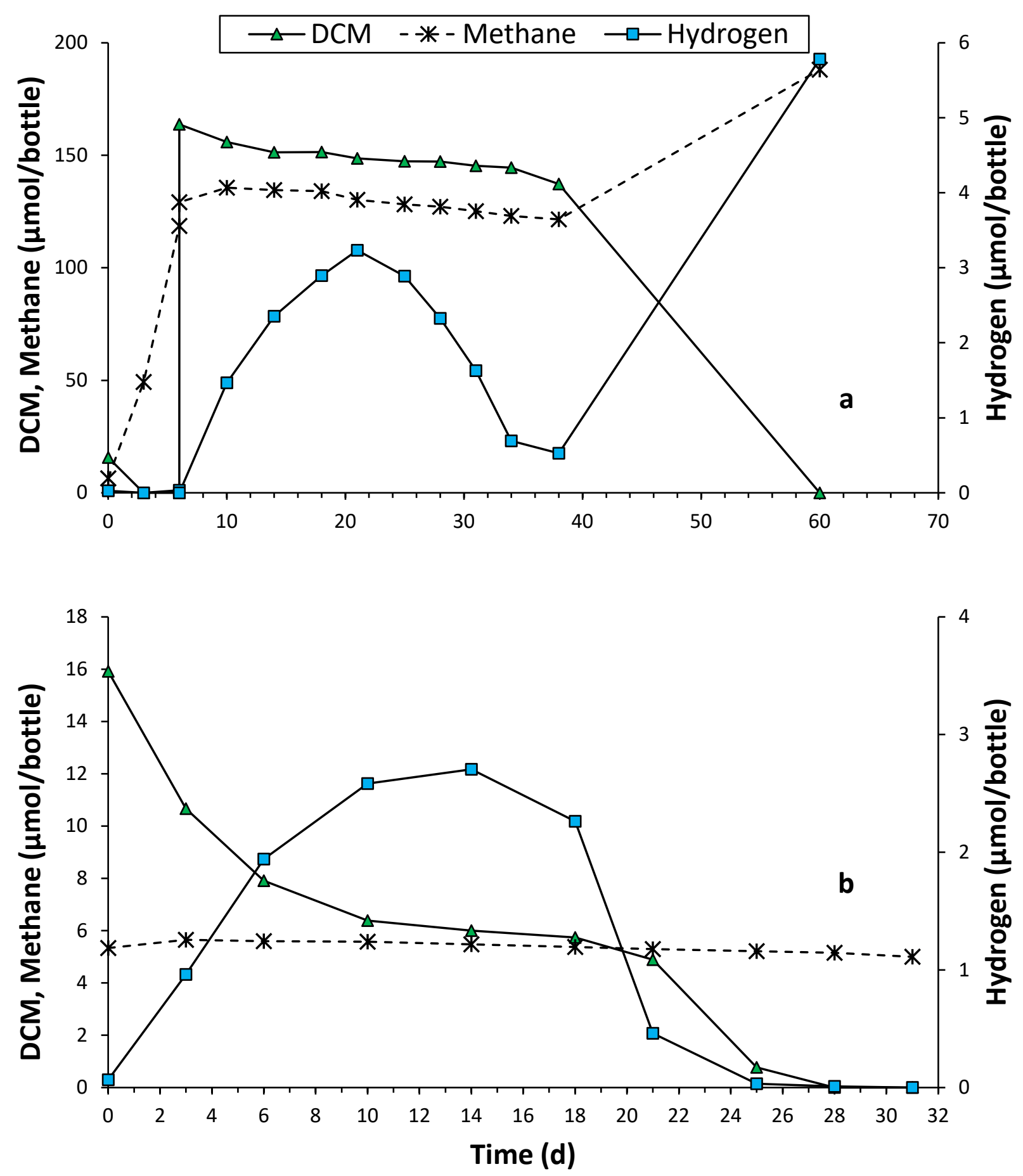

Figure 3. Biodegradation of DCM by KB-1 ${ }^{\oplus}$ Plus a) without BES added (treatment \#5); and b) with BES added (treatment \#6). Results for single bottles are shown; replicates behaved similarly (Supplementary Material). 

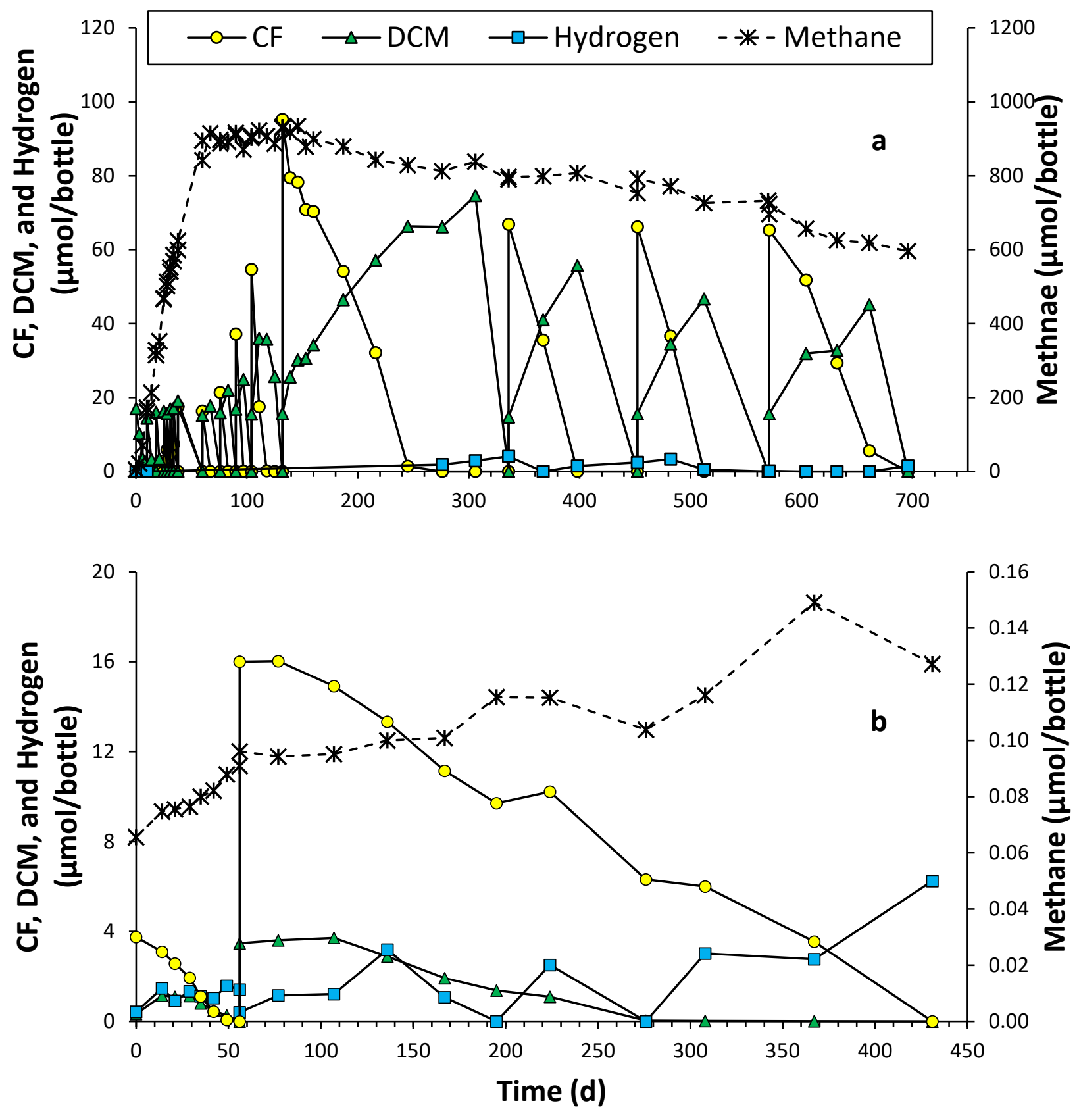

Figure 4. Biodegradation of CF by KB-1 ${ }^{\oplus}$ Plus without the addition of sodium lactate in a) treatment $\# 7$ and $\mathbf{b}$ ) treatment $\# 8$. DCM was added to treatment $\# 7$ on days $0,10,18,25,28,31$, $34,38,60,76,90,132,336,452$, and 571 . DCM was added to treatment $\# 8$ on days 0,14 , and $56(0.33,0.16$, and $3.47 \mu \mathrm{mol}$ per bottle, respectively). Results for single bottles are shown; replicates behaved similarly (Supplementary Material). 


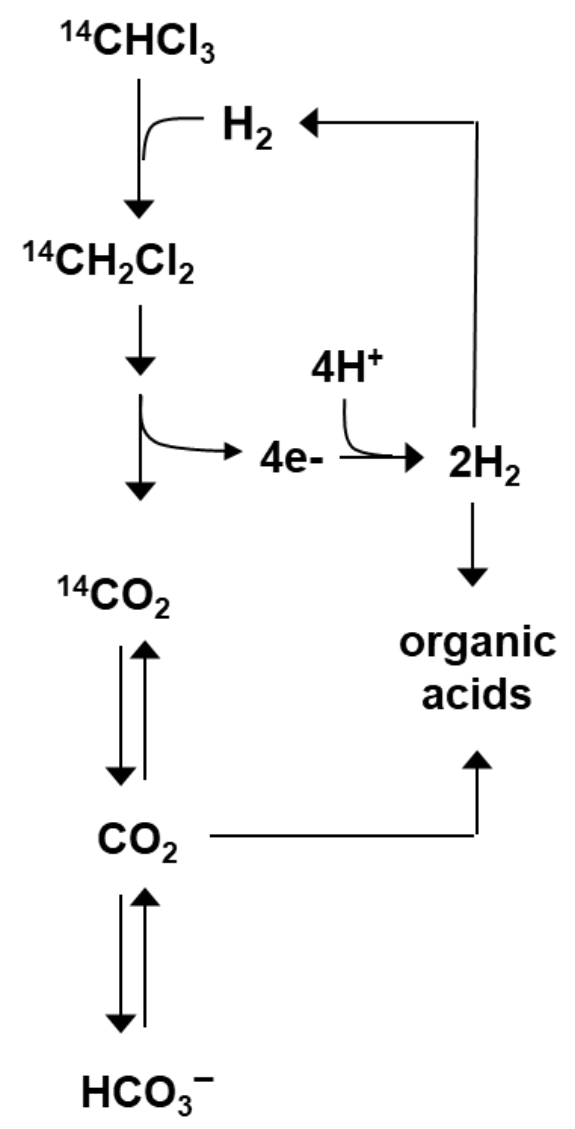

Figure 5. Proposed pathway for anaerobic biodegradation of $\mathrm{CF}$ and DCM by the KB-1 ${ }^{\circledast}$ Plus culture. 